\title{
Iron and Parkinson's disease: A systematic review and meta-analysis
}

\author{
GIOVANNI MOSTILE, CALOGERO EDOARDO CICERO, LORETTA GIULIANO, \\ MARIO ZAPPIA and ALESSANDRA NICOLETTI
}

\author{
Department of Medical Sciences, Surgical and Advanced Technologies 'G.F. Ingrassia', \\ University of Catania, I-95123 Catania, Italy
}

Received December 30, 2016; Accepted February 10, 2017

DOI: $10.3892 / \mathrm{mmr} .2017 .6386$

\begin{abstract}
A possible association between iron serum levels and Parkinson's disease (PD) using a meta-analytic approach was evaluated. A systematic MEDLINE search was conducted to identify published observational, case-control studies dealing with the association between iron blood levels and PD. In both groups, iron blood levels were extracted as means and standard deviations to calculate the standardized mean differences (SMDs) with 95\% confidence intervals (CIs). Heterogeneity of selected studies was investigated. Then, a meta-analysis was performed applying a random effects model. Possible causes of bias were also examined. A meta-regression analysis was finally conducted to investigate whether associations varied according to specified confounding factors. Of 155 studies detected by the research strategy, a total of 23 case-control studies with full available data were selected based on the adopted criteria. A small, around zero, overall SMD of -0.052 (95\% CI, -0.303-0.2) was estimated, indicating no substantial differences between groups among selected studies. High heterogeneity among studies was detected $\left(\mathrm{I}^{2}=91.42 \% ; \mathrm{p}<0.001\right)$. By performing a meta-regression analysis considering single available demographic, geographical and clinical covariates, no significant association was detected. Based on our systematic revision and meta-analysis of available case-control studies, there was not sufficient evidence supporting a possible significant association between iron serum levels and PD as compared to controls. Principal reasons should be sought in the elevated methodological heterogeneity we found among available studies. A particular attention should be paid on bias and confounding effects to limit heterogeneity among studies and to facilitate the summary of results.
\end{abstract}

Correspondence to: Professor Alessandra Nicoletti, Department of Medical Sciences, Surgical and Advanced Technologies 'G.F. Ingrassia', Via Santa Sofia 79, I-95123 Catania, Italy

E-mail: anicolet@unict.it

Key words: Parkinson's disease, iron, systematic review, meta-analysis

\section{Introduction}

Parkinson's disease (PD) is a neurological disorder with complex pathogenesis implicating both environmental and genetic factors. Epidemiological evidence suggests that chronic exposure to heavy metals such as iron, lead, manganese and their combinations can be associated with an increased risk of developing PD, since they could accumulate in the substantia nigra and generate oxidative stress (1).

Iron is an important microelement implicated in normal neuronal functioning as well as in several metabolic processes. Iron is first introduced in the body through various food products as iron salts or haemoglobin and it is then absorbed in the intestinal mucosa. Once in the enterocytes, iron is then excreted through transporter proteins (ferroportin and haephestin) in the bloodstream where it circulates attached to transferrin (2). After passing through the blood-brain barrier, iron enters the brain.

Non-physiological accumulation of iron in specific regions of the brain has been associated primarily with a heterogeneous group of diseases known as neurodegeneration with brain iron accumulation (NBIA) (3), but it has also been associated with Alzheimer's disease (4), amyotrohpic lateral sclerosis (5) and PD (6). Iron accumulation may exerts its pathogenic activity through the increase of reactive oxygen species (ROS) that then cause a wide array of damage to intracellular proteins, but there is also evidence of other mechanisms unrelated to ROS production, such as the promotion of apoptotic processes and the interaction with pathological protein aggregates found in these diseases (7).

PD is associated with extensive involvement of iron, with most extensive deposition in substantia nigra and lateral globus pallidus, as well as in dopaminergic neurons. The sources of increased iron should be searched in one or all of the following: homeostatic dysregulation, dysregulation of molecules involved in the intra and extracellular distribution of iron and aging (8).

Limited data are available concerning the levels of iron in serum/blood in PD and their relationship with the pathological process. It is not clear whether the alterations in metal homeostasis may be a cause or consequence in the pathology of the disease. However, whether metals are primary risk factors or their imbalances are consequences of pathological 
mechanisms, changes in metal ion concentration may upset the whole element homeostasis, resulting in significant imbalances in element levels in the whole system (serum, cerebrospinal fluid and brain) (9). Even more, the effect of heavy metals, including iron, derived from environmental sources such as contaminated atmosphere or food/drinking water on the pathogenesis of $\mathrm{PD}$ and their relationship with their serum levels remain still difficult to investigate (10).

Few epidemiological studies have evaluated the possible association between serum/plasma levels of iron and PD and controversial results have been reported, probably due to methodological limitations. On this ground, we conducted a systematic review and a meta-analysis of the literature to better evaluate the possible relationship between iron and PD.

We evaluated a possible significant association between iron serum levels, expression of metal exposure or result of pathological processes related to the disease, and PD as compared to controls, using a meta-analytic approach of available case-control studies.

\section{Materials and methods}

Literature search. A systematic MEDLINE search was conducted by a medical investigator without time or language restriction, to identify published observational, case-control studies dealing with the association between iron blood levels and PD. Combined text words and Medical Subject Headings (MeSH) terminology were used. Specifically, to detect available study evaluating serum trace elements in PD including iron, the following search key words and boolean operators were entered in PubMed as search strategy: (metal OR element* OR iron OR silicium OR nickel OR copper OR selenium OR zincum OR manganese OR chromium OR mercury) AND (blood or serum or plasma) AND Parkinson AND control*. Titles were scanned for relevance, identifying papers requiring further consideration. For the systematic research, a period up to September 2016 was considered.

Study selection. For the study selection, the following eligibility criteria were used considering PD as the outcome and iron blood level as exposure: i) the presence of a control group; ii) average values per group of iron blood levels together with information about methods used for the metal detection in the blood/serum and unit of measure adopted; iii) the sample sizes adopted. As additional criteria: iv) information about methods and criteria used for case-finding and control selection; v) information on demographical and geographical covariates; vi) the presence of a group-matching method by age and/or gender adopted in the selected case-control studies (if not directly available, it was subsequently tested using proper statistics); and vii) the presence of clinical information for cases characterization (i.e., disease stage or duration, motor status severity). The search results were independently assessed by a second reviewer. Disagreements were resolved through consensus among reviewers.

Data extraction and collection. The following data were extracted to be recorded in an ad hoc created collecting form: author, year of publication, measure unit adopted for the iron blood level, geographical information, setting, sample size, clinical-demographical characteristics and iron blood levels. Continuous variables were expressed as mean \pm standard deviation (SD) and min-max range, categorical variables as frequency and percent values. Specifically, iron blood levels in both groups, since continuous data, were extracted as means and SDs to calculate the standardized mean differences (SMDs) with 95\% confidence intervals (CIs).

Synthesis. Heterogeneity of selected studies was investigated using the forest plot as standardized method to display meta-analysis results and the $\mathrm{I}^{2}$ statistic (11). To estimate the association between iron blood levels and PD as compared to controls, we then performed a meta-analysis applying a random effects model as more conservative approach than its fixed effect counterpart, assuming that, in addition to sampling variation, the true effect varies between studies. In this case, the conclusion estimate is considered as the mean effect assuming that the true study effects vary (12). Possible causes of bias were also examined. Presence of publication bias or, in the presence of small studies, or bias due to low methodological quality (12), was tested graphically using the funnel plot and implemented by the fail-safe $\mathrm{N}$ calculation using the Rosenthal approach, in order to estimate the number of additional 'negative' studies that would be needed to increase the P-value for the meta-analysis to $\sim 0.05$ (13). A meta-regression analysis was also conducted to investigate whether associations varied according to specified confounding factors, in particular demographic, geographical and clinical covariates, representing possible source of heterogeneity in observational studies (12). In the case of analysis based on one covariate, either the Z-test or the Q-test (equal to $\mathrm{Z}^{2}$ ) was used to assess its relationship with effect size and thus regression model validity (14).

\section{Results}

Of 155 studies detected by the research strategy, a total of 23 case-control studies with full available data were selected based on the adopted criteria (period, 1992-2016) (Table I).

The studies were carried out in different countries [12 (52.2\%) studies in Asiatic countries, of which five in China; eight (34.8\%) studies in Europe, of which three in Italy and four in North-European countries; three (13\%) studies in the American continent, of which two in the USA].

In $21(91.3 \%)$ of the selected studies, cases selection was hospital-based, while in the other two studies it was communitybased or based on registries. Adopted sample sizes for the cases group varied among studies, with a broad range of variation (mean $\pm \mathrm{SD}, 66 \pm 52$; min-max range, 13-238). The percentage of men in PD samples was on average 57.5 \pm 11.5 (min-max range, 37.8-92.3), with an average male-to-female ratio of $1.8 \pm 2.4$ (min-max range, 0.5-12). Only 15 studies $(65.2 \%$ ) provided information on the adopted standardized diagnostic criteria for the cases selection (UK PD Society Brain Bank Clinical diagnostic criteria) (15).

Clinical information of cases were only partially provided by the adopted studies. Information on PD patients' disease duration were available in $11(47.8 \%)$ studies, with an average value per group of $5.3 \pm 2.8$ (min-max range, 2-9.5 years). Disease stage using standardized tool (the Hoehn and Yahr 
Table I. Case-control studies on blood/serum iron levels in PD and controls (CTR). Iron detection methods and iron levels among groups $(\mathrm{N}=23)$.

\begin{tabular}{|c|c|c|c|c|c|c|}
\hline Study, year (ref.) & Detection method & $\begin{array}{c}\text { Measure } \\
\text { unit }\end{array}$ & $\mathrm{PD}(\mathrm{N})$ & $\begin{array}{l}\text { PD iron level } \\
(\text { mean } \pm \text { SD) }\end{array}$ & CTR (N) & $\begin{array}{l}\text { CTR iron level } \\
(\text { mean } \pm \text { SD })\end{array}$ \\
\hline Chen and Shih, 1992 (21) & Unknown & $\mu \mathrm{g} / \mathrm{dl}$ & 15 & $95.53 \pm 33.5$ & 30 & $102.5 \pm 32.5$ \\
\hline Takahashi et al, 1994 (22) & PIXE & $\mu \mathrm{g} / \mathrm{ml}$ & 13 & $1.69 \pm 0.61$ & 14 & $1.64 \pm 0.72$ \\
\hline Logroscino et al, 1997 (23) & $\begin{array}{l}\text { Atomic absorption graphite } \\
\text { furnace micromethod }\end{array}$ & $\mu \mathrm{g} / \mathrm{dl}$ & 104 & $28.3 \pm 11.6$ & 352 & $33.9 \pm 15.2$ \\
\hline Jiménez-Jiménez et al, 1998 (24) & AAS & $\mathrm{mg} / \mathrm{l}$ & 37 & $1.01 \pm 0.33$ & 37 & $0.95 \pm 0.3$ \\
\hline Tórsdóttir et al, 1999 (25) & $\begin{array}{l}\text { Colorimetric test with } \\
\text { ferrozine ascorbic acid }\end{array}$ & $\mu \mathrm{mol} / \mathrm{l}$ & 33 & $16 \pm 4.25$ & 33 & $16 \pm 4.5$ \\
\hline Forte et al, 2004 (26) & ICP-AES & $\mu \mathrm{g} / 1$ & 26 & $1318 \pm 481$ & 13 & $1136 \pm 393$ \\
\hline Hedge et al, 2004 (9) & ICP-AES & $\mu \mathrm{mol} / \mathrm{ml}$ & 27 & $0.02 \pm 0.004$ & 25 & $0.023 \pm 0.009$ \\
\hline Qureshi et al, 2006 (27) & AAS & $\mathrm{mg} / \mathrm{ml}$ & 17 & $1.02 \pm 0.11$ & 21 & $1.16 \pm 0.05$ \\
\hline Annanmaki et al, 2007 (28) & $\begin{array}{l}\text { Spectrophotometric } \\
\text { reaction with ferrozine }\end{array}$ & $\mu \mathrm{mol} / 1$ & 40 & $18.2 \pm 5.5$ & 29 & $20.5 \pm 6.3$ \\
\hline Squitti et al, 2007 (29) & AAS & unknown & 65 & $91.1 \pm 18.1$ & 52 & $84.5 \pm 18.1$ \\
\hline Gellein et al, 2008 (30) & HR-ICP-MS & $\mu \mathrm{g} / 1$ & 33 & $1275 \pm 551$ & 99 & $1146 \pm 463$ \\
\hline Ahmed and Santosh, 2010 (31) & ICP-AES and ICP-MS & $\mu \mathrm{g} / \mathrm{dl}$ & 45 & $110.4 \pm 0.6$ & 42 & $123 \pm 8$ \\
\hline Fukushima et al, 2010 (10) & ICP-AES & $\mu \mathrm{g} / \mathrm{ml}$ & 82 & $2 \pm 0.83$ & 82 & $1.5 \pm 0.78$ \\
\hline Fukushima et al, 2011 (32) & ICP-AES & $\mu \mathrm{g} / \mathrm{ml}$ & 71 & $1.95 \pm 0.85$ & 71 & $1.44 \pm 0.77$ \\
\hline Madenci et al, 2012 (33) & Unknown & unknown & 60 & $74.6 \pm 29.3$ & 42 & $74.8 \pm 27.1$ \\
\hline Farhoudi et al, 2012 (34) & $\begin{array}{l}\text { Unspecified biochemical } \\
\text { methods }\end{array}$ & $\mathrm{mg} / \mathrm{dl}$ & 50 & $70.22 \pm 25.18$ & 50 & $67.62 \pm 39.53$ \\
\hline Fukushima et al, 2013 (35) & ICP-AES & $\mu \mathrm{g} / \mathrm{ml}$ & 58 & $2.1 \pm 0.84$ & 81 & $1.51 \pm 0.78$ \\
\hline Zhao et al, 2013 (36) & $\begin{array}{l}\text { Fast sequential atomic } \\
\text { absorption spectroscopy }\end{array}$ & $\mu \mathrm{g} / 1$ & 238 & $1656 \pm 749$ & 302 & $1470 \pm 648$ \\
\hline Kumudini et al, 2014 (37) & ICP-MS & $\mathrm{ng} / \mathrm{ml}$ & 150 & $554.4 \pm 123.8$ & 175 & $421.7 \pm 126.1$ \\
\hline Hu et al, 2015 (38) & ELISA & $\mathrm{nmol} / \mathrm{ml}$ & 102 & $4.124 \pm 1.064$ & 31 & $4.192 \pm 1.054$ \\
\hline Costa-Mallen et al, 2015 (39) & Unknown & $\mu \mathrm{g} / 100 \mathrm{ml}$ & 128 & $83.28 \pm 29.46$ & 226 & $94 \pm 34.14$ \\
\hline Madeiros et al, 2016 (40) & $\begin{array}{l}\text { Standard methods } \\
\text { on cobas mira }\end{array}$ & $\mu \mathrm{g} / \mathrm{dl}$ & 40 & $67.5 \pm 18.89$ & 46 & $78 \pm 18.15$ \\
\hline Mariani et al, 2016 (41) & Ferene & $\mu \mathrm{g} / \mathrm{dl}$ & 92 & $79 \pm 34$ & 112 & $86.1 \pm 34.9$ \\
\hline
\end{tabular}

Takahashi et al: 'untreated PD' and 'elderly controls' have been selected (age difference, p=0.915); Logroscino et al: matching done, but no data available; Tórsdóttir et al: SD estimated from range; Forte et al: PD HY stage, 1-3. 'Serum' (not 'blood') concentrations were selected; Hedge et al: only 'early PD group' considered: best age- $(\mathrm{p}=0.287)$ and gender- $(\mathrm{p}=0.991)$ matching; Qureshi et al: PD in 'on' phase selected: best age-matching ( $\mathrm{p}=0.08)$; Squitti et al: two regions, only 'Valcamonica' region selected for PD and CTR (highest N); Gellein et al: preclinical PD group has been selected. Date for PD age, 1997; Fukushima et al: PD, 'no depression' group was selected; age- ( $\mathrm{p}=0.711)$ and gender- (p=0.632) matched; Hu et al: PD, no RBD group. HY and disease duration as median (range). Mean and SD were estimated; Madeiros et al: medians and ranges provided. Means and SD were estimated. CTR from Santa Maria, same state; Mariani et al: medians and ranges provided. Means and SD were estimated. Age and gender matching (p<0.001). PD, Parkinson's disease; PIXE, Particle-Induced X-ray Emission; AAS, atomic absorption spectrophotometer; ICP-AES, inductively coupled plasma atomic emission spectrometry; HR-ICP-MS, high resolution inductively coupled plasma mass spectrometry; ELISA, enzyme-linked immunosorbent assay.

scale) (16) was provided in three (13\%) studies (average value per group of $2 \pm 1$; min-max range, 1-2.9). Standardized information on patients' motor status using the Unified PD Rating Scale (UPDRS) (17) total score was provided in seven $(30.4 \%)$ studies, with an average value per group of $32.8 \pm 11.4$ (min-max range, 18.8-45.1) (Table II).

Concerning the control groups, selection was hospital-based in $19(82.6 \%)$ studies, while in the other three studies it was community-based and in a single study based on registries. An age and gender group-matching strategy was adopted in $16(69.6 \%)$ studies, while a group-matching only by age was adopted in five $(21.7 \%)$ studies. One study did not adopt any group-matching strategy. No data were provided in one study (Table III).
Iron serum levels (using different units of measure) in both groups and the different methods of detection adopted are shown in Table I. A meta-analysis was performed on all the 23 studies included. Results are summarized as forest plot in Fig. 1. A small, around zero, overall SMD of -0.052 (95\% CI, -0.303-0.2) was estimated, indicating no substantial differences between groups among selected studies. $\mathrm{I}^{2}$ statistic revealed high heterogeneity among studies $\left(\mathrm{I}^{2}=91.42 \%\right.$; $\left.<<0.001\right)$; it means that $\sim 91 \%$ of the observed variance comes from real differences between studies and can potentially be explained by study-level covariates.

Asymmetry in funnel plot was consistent with the presence of publication bias in favor of positive results, showing also that studies were principally allocated in bottom of the 
Table II. Case-control studies on blood/serum iron levels in PD and controls (CTR). PD group characteristics.

\begin{tabular}{|c|c|c|c|c|c|c|}
\hline Study, year (ref.) & $\mathrm{PD}(\mathrm{N})$ & PD source & $\begin{array}{l}\text { Diagnostic } \\
\text { criteria }\end{array}$ & $\begin{array}{l}\text { PD men } \\
(\mathrm{N}, \%)\end{array}$ & $\mathrm{PD} \mathrm{M} / \mathrm{F}$ ratio & $\begin{array}{c}\text { PD age } \\
(\text { mean } \pm \text { SD })\end{array}$ \\
\hline Chen and Shih, 1992 (21) & 15 & Hospital & Not specified & Not specified & Not specified & Not specified \\
\hline Takahashi et al, 1994 (22) & 13 & Hospital & Not specified & Not specified & Not specified & $61.2 \pm 10.3$ \\
\hline Logroscino et al, 1997 (23) & 104 & Community & UK Brain Bank & Not specified & Not specified & Not specified \\
\hline Jiménez-Jiménez et al, 1998 (24) & 37 & Hospital & UK Brain Bank & $14(37.8)$ & 0.61 & $65.7 \pm 8.8$ \\
\hline Tórsdóttir et al, 1999 (25) & 33 & Hospital & Not specified & $18(54.5)$ & 1.2 & $67 \pm 8.5$ \\
\hline Forte et al, 2004 (26) & 26 & Hospital & UK Brain Bank & $24(92.3)$ & 12 & $64.9 \pm 10.8$ \\
\hline Hedge et al, 2004 (9) & 27 & Hospital & Not specified & $14(51.8)$ & 1.08 & $57.1 \pm 5.2$ \\
\hline Qureshi et al, 2006 (27) & 17 & Hospital & UK Brain Bank & $10(58.8)$ & 1.43 & $70 \pm 15$ \\
\hline Annanmaki et al, 2007 (28) & 40 & Hospital & UK Brain Bank & $23(58)$ & 1.35 & $60.8 \pm 6.5$ \\
\hline Squitti et al, 2007 (29) & 65 & Hospital & Not specified & $34(52.3)$ & 1.1 & $67.9 \pm 7.1$ \\
\hline Gellein et al, 2008 (30) & 33 & Hospital & UK Brain Bank & $16(48.5)$ & 0.94 & $61.1 \pm 9.1$ \\
\hline Ahmed and Santosh, 2010 (31) & 45 & Hospital & Not specified & $26(57.8)$ & 1.37 & $57.6 \pm 9.1$ \\
\hline Fukushima et al, 2010 (10) & 82 & Hospital & UK Brain Bank & $47(57.3)$ & 1 & $63.9 \pm 9.4$ \\
\hline Fukushima et al, 2011 (32) & 71 & Hospital & UK Brain Bank & $41(57.7)$ & 1.37 & $63.7 \pm 9.7$ \\
\hline Madenci et al, 2012 (33) & 60 & Hospital & UK Brain Bank & $33(55)$ & 1.22 & $68.5 \pm 9.2$ \\
\hline Farhoudi et al, 2012 (34) & 50 & Hospital & Not specified & $28(56)$ & 1.27 & $64.5 \pm 10.2$ \\
\hline Fukushima et al, 2013 (35) & 58 & Hospital & UK Brain Bank & $36(62.1)$ & 1.64 & $64.3 \pm 9.4$ \\
\hline Zhao et al, 2013 (36) & 238 & Hospital & UK Brain Bank & $121(50.8)$ & 1.03 & $66.6 \pm 11.3$ \\
\hline Kumudini et al, 2014 (37) & 150 & Hospital & Not specified & $107(71.3)$ & 2.49 & $55.7 \pm 10.6$ \\
\hline Hu et al, 2015 (38) & 102 & Hospital & UK Brain Bank & $47(46.1)$ & 0.46 & $56.3 \pm 13.4$ \\
\hline Costa-Mallen et al, 2015 (39) & 128 & Registry & UK Brain Bank & $88(68.7)$ & 2.2 & 69 \\
\hline Madeiros et al, 2016 (40) & 40 & Hospital & UK Brain Bank & $18(45)$ & 0.82 & $69.95 \pm 12.3$ \\
\hline Mariani et al, 2016 (41) & 92 & Hospital & UK Brain Bank & $62(67.4)$ & 2.07 & $70 \pm 11.25$ \\
\hline
\end{tabular}

Table III. Case-control studies on blood/serum iron levels in PD and controls (CTR). CTR group characteristics.

\begin{tabular}{|c|c|c|c|c|c|c|c|}
\hline Study, year (ref.) & CTR $(\mathrm{N})$ & $\begin{array}{l}\text { CTR } \\
\text { source }\end{array}$ & $\begin{array}{l}\text { CTR men } \\
(\mathrm{N}, \%)\end{array}$ & $\begin{array}{c}\text { CTR } \\
\mathrm{M} / \mathrm{F} \text { ratio }\end{array}$ & $\begin{array}{c}\text { CTR age } \\
(\text { mean } \pm \text { SD })\end{array}$ & $\begin{array}{c}\text { Age } \\
\text { matching }\end{array}$ & $\begin{array}{l}\text { Gender } \\
\text { matching }\end{array}$ \\
\hline Chen and Shih, 1992 (21) & 30 & Hospital & Not specified & Not specified & Not specified & Not specified & Not specified \\
\hline Takahashi et al, 1994 (22) & 14 & Hospital & Not specified & Not specified & $60.8 \pm 8.9$ & Yes & Not specified \\
\hline Logroscino et al, 1997 (23) & 352 & Community & Not specified & Not specified & Not specified & Yes & No \\
\hline Jiménez-Jiménez et al, 1998 (24) & 37 & Hospital & $16(43.2)$ & 0.76 & $62.4 \pm 17.8$ & Yes & Yes \\
\hline Tórsdóttir et al, 1999 (25) & 33 & Hospital & Not specified & Not specified & Not specified & Yes & Yes \\
\hline Forte et al, 2004 (26) & 13 & Hospital & $6(46.1)$ & 0.86 & $63.8 \pm 13.7$ & Yes & No \\
\hline Hedge et al, 2004 (9) & 25 & Hospital & $13(52)$ & 1.08 & $55.4 \pm 6.4$ & Yes & Yes \\
\hline Qureshi et al, 2006 (27) & 21 & Hospital & $13(61.9)$ & 1.63 & $62 \pm 11$ & Yes & Yes \\
\hline Annanmaki et al, 2007 (28) & 29 & Hospital & $13(45)$ & 0.81 & $60.2 \pm 5.1$ & Yes & Yes \\
\hline Squitti et al, 2007 (29) & 52 & Hospital & $32(61.5)$ & 1.6 & $71.1 \pm 8.5$ & Yes & Yes \\
\hline Gellein et al, 2008 (30) & 99 & Community & $48(48.5)$ & 0.94 & Not specified & Yes & Yes \\
\hline Ahmed and Santosh, 2010 (31) & 42 & Hospital & $25(59.9)$ & 1.47 & $55.6 \pm 3.2$ & Yes & Yes \\
\hline Fukushima et al, 2010 (10) & 82 & Hospital & $47(57.3)$ & 1 & $63.6 \pm 9.3$ & Yes & Yes \\
\hline Fukushima et al, 2011 (32) & 71 & Hospital & $41(57.7)$ & 1.37 & $63.4 \pm 9.7$ & Yes & Yes \\
\hline Madenci et al, 2012 (33) & 42 & Hospital & $22(52.4)$ & 1.1 & $66.9 \pm 8.3$ & Yes & Yes \\
\hline Farhoudi et al, 2012 (34) & 50 & Hospital & $25(50)$ & 1 & $63.5 \pm 9.8$ & Yes & Yes \\
\hline Fukushima et al, 2013 (35) & 81 & Hospital & $47(58)$ & 1.38 & $63.7 \pm 9.4$ & Yes & Yes \\
\hline Zhao et al, 2013 (36) & 302 & Hospital & $153(50.7)$ & 1.03 & $65.6 \pm 12.2$ & Yes & Yes \\
\hline Kumudini et al, 2014 (37) & 175 & Hospital & $120(70.6)$ & 2.18 & $53.7 \pm 10.9$ & Yes & Yes \\
\hline Hu et al, 2015 (38) & 31 & Hospital & Not specified & Not specified & Not specified & Yes & Not specified \\
\hline Costa-Mallen et al, 2015 (39) & 226 & Registry & $104(46)$ & 0.85 & 62.6 & Yes & Not specified \\
\hline Madeiros et al, 2016 (40) & 46 & Community & $19(41)$ & 0.7 & $62.3 \pm 10.2$ & Yes & Yes \\
\hline Mariani et al, 2016 (41) & 112 & Hospital & $40(35.8)$ & 0.56 & $62 \pm 14$ & No & No \\
\hline
\end{tabular}




\begin{tabular}{|c|c|c|c|}
\hline Studies & Est & imate $(958$ & (C.I.) \\
\hline Chen, 1992 & -0.209 & $(-0.830$, & $0.413)$ \\
\hline Takahashi, 1994 & 0.072 & $(-0.683$, & $0.828)$ \\
\hline Logroscino, 1997 & -0.387 & $(-0.607$ & $-0.166)$ \\
\hline Jiménez-Jiménez, 1998 & 0.188 & $(-0.268$, & $0.645)$ \\
\hline Tórsdóttir, 1999 & 0.000 & $(-0.483$ & $0.483)$ \\
\hline Forte, 2004 & 0.392 & $(-0.279$ & $1.064)$ \\
\hline Hedge, 2004 & -0.430 & $(-0.980$ & $0.120)$ \\
\hline Qureshi, 2006 & -1.666 & $(-2.407$ & -0.925 \\
\hline Annanmaki, 2007 & -0.389 & $(-0.871$, & $0.094)$ \\
\hline Squitti, 2007 & 0.362 & $(-0.005$ & $0.730)$ \\
\hline Gellein, 2008 & 0.264 & $(-0.131$ & $0.659)$ \\
\hline Ahmed, 2010 & -2.241 & $(-2.777$, & $-1.705)$ \\
\hline Fukushima, 2010 & 0.618 & $(0.305$ & $0.931)$ \\
\hline Fukushima, 2011 & 0.625 & $(0.289$, & $0.962)$ \\
\hline Madenci, 2012 & -0.007 & $(-0.401$ & 0.387 \\
\hline Farhoudi, 2012 & 0.078 & $(-0.314$, & $0.470)$ \\
\hline Fukushima, 2013 & 0.728 & (0.381, & $1.076)$ \\
\hline Zhao, 2013 & 0.268 & 10.097 & $0.438)$ \\
\hline Kumudini, 2014 & 1.059 & 10.826, & $1.292)$ \\
\hline $\mathrm{Hu}, 2015$ & -0.064 & $(-0.466$, & $0.338)$ \\
\hline Costa-Mallen, 2015 & -0.329 & $(-0.547$ & -0.111 \\
\hline Madeiros, 2016 & -0.563 & $(-0.995$ & $-0.131)$ \\
\hline Mariani, 2016 & -0.205 & $(-0.482$, & $0.071)$ \\
\hline Jverall $\left(I^{\wedge} 2=91.42 \%, P<0.001\right)$ & - .00 & $(-0.303$, & $0.200)$ \\
\hline
\end{tabular}

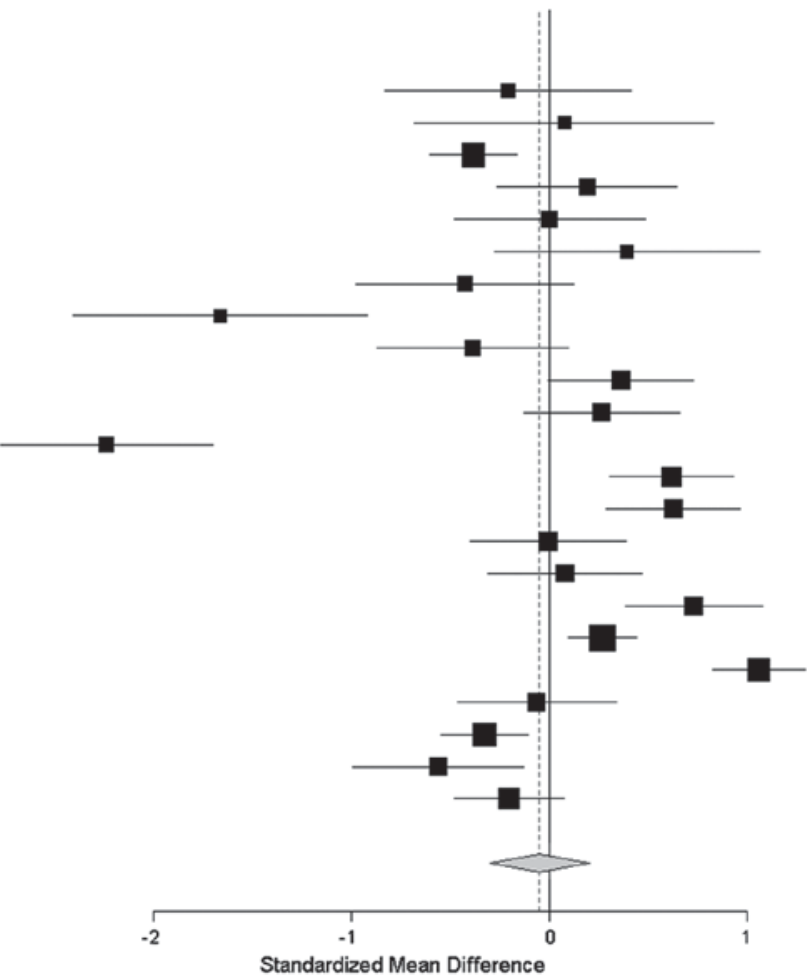

Figure 1. Iron serum concentration in PD and controls (N=23). Forest plot with SMDs (95\% CI) (random-effect method). PD, Parkinson's disease; SMDs, standardized mean differences; CI, confidence interval.

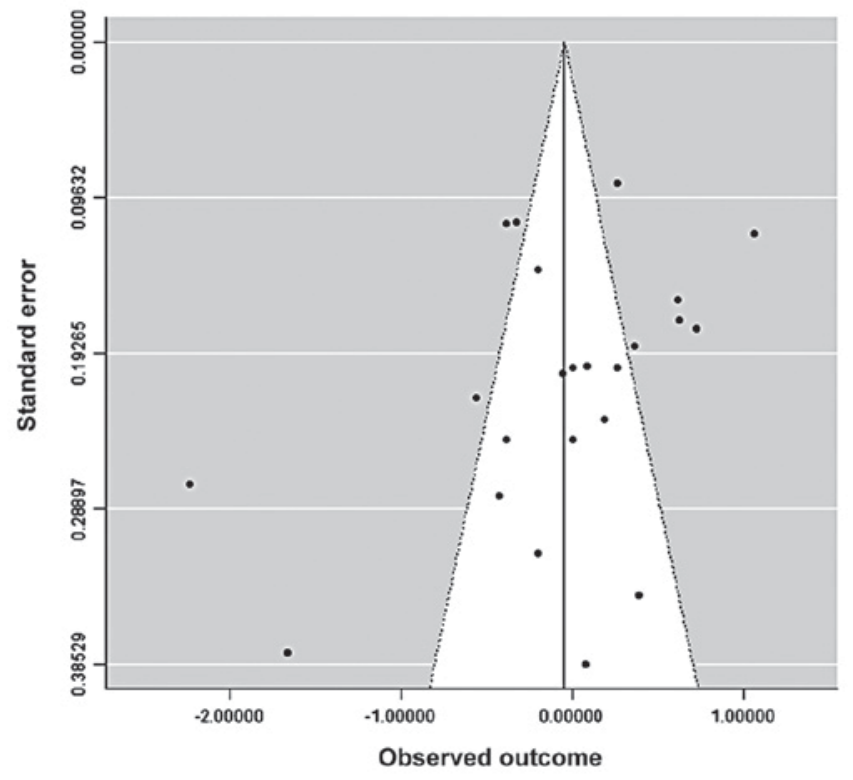

Figure 2. Funnel plot ( $=23)$.

graph indicating a broad range of standard error of the effect measures among studies due to their small sample size (Fig. 2). The fail-safe $\mathrm{N}$ calculation was equal to zero (observed significance level, 0.319; target significance level, 0.05).

A meta-regression analysis was then performed considering single demographic, geographical and clinical covariates using a random-effects model. The regression coefficient for latitude was 0.003 , which means that every one degree of latitude corresponds to an increase of 0.003 units in effect size, even if this was not statistically significant $(Z=0.457$,
$\mathrm{Q}=0.209, \mathrm{p}=0.648$ ). Regression coefficients for longitude and altitude were respectively $0.002(\mathrm{Z}=1.175, \mathrm{Q}=1.38, \mathrm{p}=0.240)$ and $0(\mathrm{Z}=0.721, \mathrm{Q}=0.52, \mathrm{p}=0.471)$, both not statistically significant (Fig. 3).

Demographical covariates, based on the assumption that most of studies were group-matched by age and gender, the regression coefficient for PD age was $-0.01(\mathrm{~N}=21, \mathrm{Z}=-0.269$, $\mathrm{Q}=0.072, \mathrm{p}=0.788)$ while for the $\mathrm{PD}$ male-to-female ratio was $0.049(\mathrm{~N}=20, \mathrm{Z}=0.66, \mathrm{Q}=0.436, \mathrm{p}=0.509)$, both not statistically significant (Fig. 3).

When we looked at the clinical characteristics of selected groups of cases, we found a regression coefficient for disease duration of $0.035(\mathrm{~N}=11, \mathrm{Z}=0.607, \mathrm{Q}=0.368, \mathrm{p}=0.544)$ and for the UPDRS total score of $0.017(\mathrm{~N}=7, \mathrm{Z}=1.28, \mathrm{Q}=1.64$, $\mathrm{p}=0.201)$, both not statistically significant.

\section{Discussion}

Iron and its deregulated homeostasis have been proposed to have a role in the pathogenesis of PD because of its pro-oxidants characteristics that may lead to ROS generation via Fenton and Haber-Weiss reactions. However, epidemiological evidence concerning the possible association between iron and PD remains still controversial.

In this systematic revision and meta-analysis, we searched for a possible association between serum iron levels and PD, as compared to controls. Based on selected case-control studies, we did not find a significant pooled mean difference between groups. Our results are in agreement with a previous meta-analysis by Mariani et al (18) demonstrating no variation of metal concentrations in serum between PD patients and healthy controls (SMD, -0.45; 95\% CI, -0.98-0.08), probably due to 

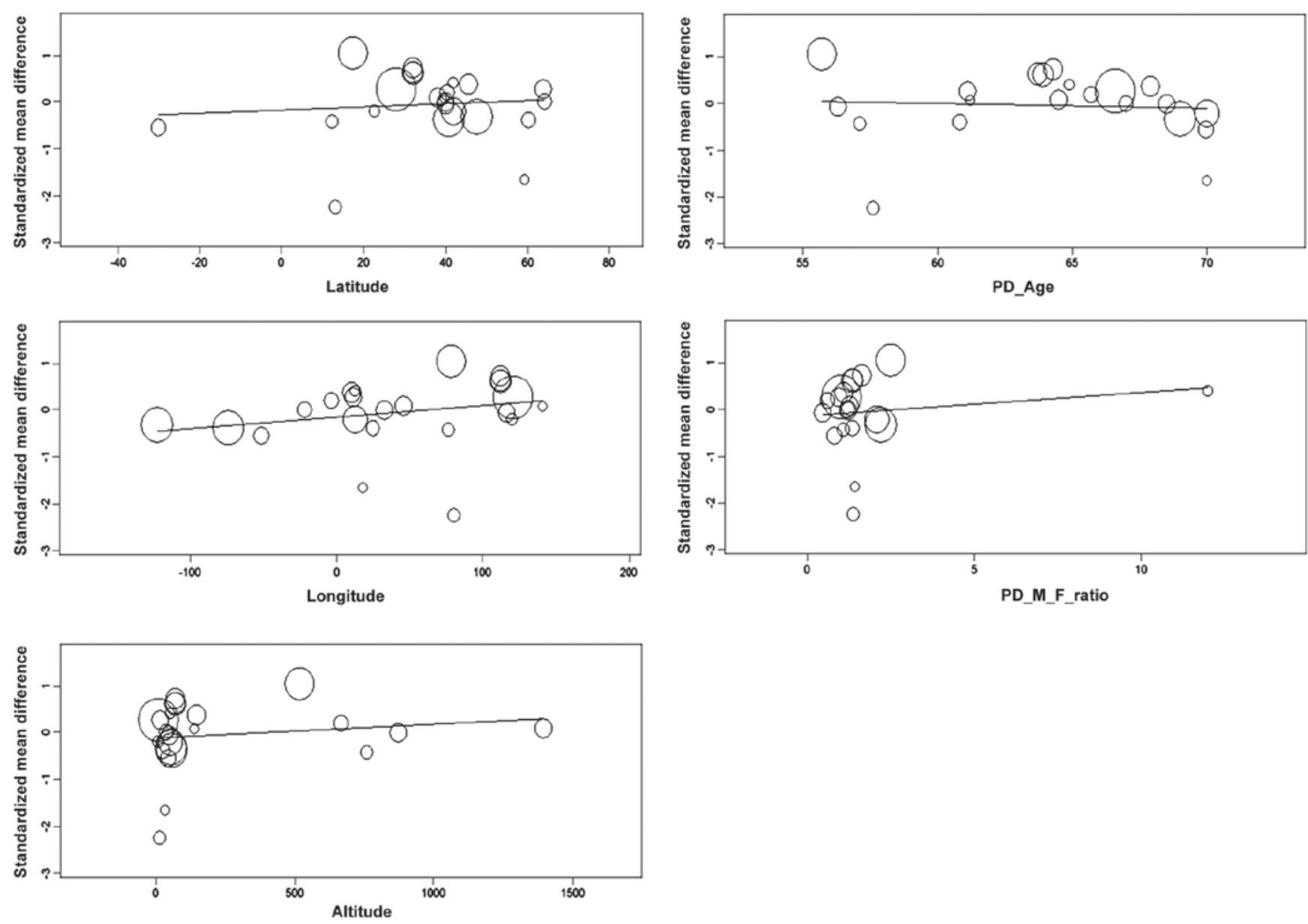

Figure 3. Meta-regression plots. Geographical and demographic covariates (random-effect method).

the high heterogeneity among evaluated studies $\left(\mathrm{I}^{2}=93.4 \%\right.$; $\mathrm{p}<0.001)$. Evaluation of a second meta-analysis based on 11 selected studies showed instead overall higher iron serum levels in PD patients when compared to controls (SMD, 0.97; 95\% CI, 0.18-0.37), even though a significant heterogeneity was also found among studies $\left(\mathrm{I}^{2}=96.5 \%\right.$; $\mathrm{p}<0.001)(19)$.

We confirmed a high level of heterogeneity among evaluated studies, as expression of the overall small-sampled, methodologically-limited studies available in literature which are insufficient to provide practical evidence; for instance, most of the selected studies had a hospital-based design, which does not permit to exclude a possible selection bias. Moreover, the presence of publication bias could have lead to an underestimation of possible negative results.

Heterogeneity among studies can be related to several issues: the different approaches in finding and selection of cases and controls; the lack of an adequate matching strategy between groups; the use of an inadequate sample size for obtaining statistically-relevant results; the different methods used for iron detection in blood samples; the lack of corrected analysis for possible confounders including comorbidities affecting iron serum levels. These issues justified the choice of a random-effects approach for meta-analysis of data.

We performed a meta-regression analysis considering single available demographic, geographical and clinical covariates, all potential confounders affecting our pooled results. However, no significant association was detected.

Limits of the present meta-analysis are the same as those related to other meta-analysis performed using observational, case-control studies as target. In particular, the appropriate control of confounding factors is of fundamental importance in the analysis and interpretation of observational studies. The presence of other types of bias, for example recall bias, should represent additional concerns (12). Even more, we focused our selection on studies evaluating serum iron levels and not other biological fluids (i.e., urine or cerebrospinal fluid) or tissues (i.e., hair), limiting results interpretation. Furthermore, it should be underlined that in case-control studies evaluating a possible association between metals and PD by detecting levels of metals in biological fluids, these measurements often show just the actual iron homeostasis, lacking a clear history of exposure to high levels of this metal (20). Finally, even if a systematical approach has been used for the search strategy, we cannot exclude that some data were missed affecting the study results.

In conclusion, based on our systematic review and meta-analysis of available case-control studies, we can state there are still not sufficient evidence supporting higher or lower serum levels of iron in PD patients as compared to controls, assuming this may be related to metal exposure or pathological processes in such subjects. Principal reasons should be sought in the elevated methodological heterogeneity we found among 
available studies. A particular attention should be paid on bias and confounding effects to limit heterogeneity among studies and to facilitate the summary of the results.

\section{References}

1. Chin-Chan M, Navarro-Yepes J and Quintanilla-Vega B: Environmental pollutants as risk factors for neurodegenerative disorders: alzheimer and Parkinson diseases. Front Cell Neurosci 9: 124, 2015.

2. Fuqua BK, Vulpe $C D$ and Anderson GJ: Intestinal iron absorption. J Trace Elem Med Biol 26: 115-119, 2012.

3. Schneider SA, Hardy J and Bhatia KP: Syndromes of neurodegeneration with brain iron accumulation (NBIA): an update on clinical presentations, histological and genetic underpinnings, and treatment considerations. Mov Disord 27: 42-53, 2012.

4. Smith MA, Zhu X, Tabaton M, Liu G, McKeel DW Jr, Cohen ML, Wang X, Siedlak SL, Dwyer BE, Hayashi T, et al: Increased iron and free radical generation in preclinical alzheimer disease and mild cognitive impairment. J Alzheimers Dis 19: 363-372, 2010.

5. Kasarskis EJ, Tandon L, Lovell MA and Ehmann WD: Aluminum, calcium, and iron in the spinal cord of patients with sporadic amyotrophic lateral sclerosis using laser microprobe mass spectroscopy: a preliminary study. J Neurol Sci 130: 203-208, 1995

6. Dexter DT, Wells FR, Agid F, Agid Y, Lees AJ, Jenner P and Marsden CD: Increased nigral iron content in postmortem Parkinsonian brain. Lancet 2: 1219-1220, 1987.

7. Ward RJ, Zucca FA, Duyn JH, Crichton RR and Zecca L: The role of iron in brain ageing and neurodegenerative disorders Lancet Neurol 13: 1045-1060, 2014.

8. Jellinger KA: The relevance of metals in the pathophysiology of neurodegeneration, pathological considerations. Int Rev Neurobiol 110: 1-47, 2013.

9. Hegde ML, Shanmugavelu P, Vengamma B, Rao TS, Menon RB, Rao RV and Rao KS: Serum trace element levels and the complexity of inter-element relations in patients with Parkinson's disease. J Trace Elem Med Biol 18: 163-171, 2004.

10. Fukushima T, Tan X, Luo Y and Kanda H: Relationship between blood levels of heavy metals and Parkinson's disease in China. Neuroepidemiology 34: 18-24, 2010.

11. Higgins JP, Thompson SG, Deeks JJ and Altman DG: Measuring inconsistency in meta-analyses. BMJ 327: 557-560, 2003.

12. Kirkwood BR and Sterne JAC (eds): Essential Medical Statistics. 2nd edition. Blackwell Publishing, Oxford, 2003.

13. Rosenthal R: The 'file drawer problem' and tolerance for null results. Psychol Bull 86: 638-641, 1979.

14. Borenstein M, Hedges LV, Higgins JPT and Rothstein HR (eds): Introduction to Meta-Analysis. Wiley, Chichester, UK, 2009.

15. Hughes AJ, Daniel SE, Kilford L and Lees AJ: Accuracy of clinical diagnosis of idiopathic Parkinson's disease: a clinico-pathological study of 100 cases. J Neurol Neurosurg Psychiatry 55: 181-184, 1992.

16. Hoehn MM and Yahr MD: Parkinsonism: onset, progression and mortality. Neurology 17: 427-442, 1967.

17. Fahn S and Elton RL: Unified Parkinson's disease rating scale. In: Recent Developments in Parkinson's Disease. Fahn S, Marsden CD, Calne DB and Goldstein M (eds). Vol 2. Macmillan, Florham Park, NJ, pp153-163, 1987.

18. Mariani S, Ventriglia M, Simonelli I, Donno S, Bucossi S, Vernieri F, Melgari JM, Pasqualetti P, Rossini PM and Squitti R: $\mathrm{Fe}$ and $\mathrm{Cu}$ do not differ in Parkinson's disease: a replication study plus meta-analysis. Neurobiol Aging 34: 632-633, 2013.

19. Jiao J, Guo H, He Y, Wang J, Yuan J and Hu W: Meta-analysis of the association between serum iron levels and Parkinson's disease: evidence from 11 publications. Brain Res 1646: 490-493, 2016.

20. Sahin C, Pala C, Kaynar L, Torun YA, Cetin A, Kurnaz F, Sivgin S and Sahin FS: Measurement of hair iron concentration as a marker of body iron content. Biomed Rep 3: 383-387, 2015.

21. Chen WH and Shih PY: The serum ferrokinetics in Parkinson's disease. Gaoxiong Yi Xue Ke Xue Za Zhi 8: 581-584, 1992 (In Chinese).

22. Takahashi S, Takahashi J, Osawa N, Abe T, Yonezawa H, Sera K and Tohgi H: Trace elements analysis of serum and cerebrospinal fluid with PIXE-effect of age and changes in Parkinsonian patients. Nippon Ronen Igakkai Zasshi 31: 865-871, 1994 (In Japanese).
23. Logroscino G, Marder K, Graziano J, Freyer G, Slavkovich V, Lolacono N, Cote L and Mayeux R: Altered systemic iron metabolism in Parkinson's disease. Neurology 49: 714-717, 1997.

24. Jiménez-Jiménez FJ, Molina JA, Aguilar MV, Meseguer I, Mateos-Vega CJ, González-Muñoz MJ, de Bustos F, Martínez-Salio A, Ortí-Pareja M, Zurdo M, et al: Cerebrospinal fluid levels of transition metals in patients with Parkinson's disease. J Neural Transm Vienna 105: 497-505, 1998.

25. Tórsdóttir G, Kristinsson J, Sveinbjörnsdóttir S, Snaedal J and Jóhannesson T: Copper, ceruloplasmin, superoxide dismutase and iron parameters in Parkinson's disease. Pharmacol Toxicol 85: 239-243, 1999.

26. Forte G, Bocca B, Senofonte O, Petrucci F, Brusa L, Stanzione P, Zannino S, Violante N, Alimonti A and Sancesario G: Trace and major elements in whole blood, serum, cerebrospinal fluid and urine of patients with Parkinson's disease. J Neural Transm Vienna 111: 1031-1040, 2004.

27. Qureshi GA, Qureshi AA, Memon SA and Parvez SH: Impact of selenium, iron, copper and zinc in on/off Parkinson's patients on L-dopa therapy. J Neural Transm (Suppl) 71: 229-236, 2006.

28. Annanmaki T, Muuronen A and Murros K: Low plasma uric acid level in Parkinson's disease. Mov Disord 22: 1133-1137, 2007.

29. Squitti R, Gorgone G, Binetti G, Ghidoni R, Pasqualetti P, Draicchio F, Albini E, Benedetti L, Lucchini R and Rossini PM: Metals and oxidative stress in Parkinson's disease from industrial areas with exposition to environmental toxins or metal pollution. G Ital Med Lav Ergon 29 (Suppl 3): 294-296, 2007 (In Italian).

30. Gellein K, Syversen T, Steinnes E, Nilsen TI, Dahl OP, Mitrovic S, Duraj D and Flaten TP: Trace elements in serum from patients with Parkinson's disease: a prospective case-control study: the Nord-Trøndelag health study (HUNT). Brain Res 1219: 111-115, 2008.

31. Ahmed SS and Santosh W: Metallomic profiling and linkage map analysis of early Parkinson's disease: a new insight to aluminum marker for the possible diagnosis. PLoS One 5: e11252, 2010.

32. Fukushima T, Tan X, Luo Y and Kanda H: Serum vitamins and heavy metals in blood and urine, and the correlations among them in Parkinson's disease patients in China. Neuroepidemiology 36: 240-244, 2011.

33. Madenci G, Bilen S, Arli B, Saka M and Ak F: Serum iron, vitamin B12 and folic acid levels in Parkinson's disease. Neurochem Res 37: 1436-1441, 2012.

34. Farhoudi M, Taheraghdam A, Farid GA, Talebi M, Pashapou A, Majidi $\mathrm{J}$ and Goldust M: Serum iron and ferritin level in idiopathic Parkinson. Pak J Biol Sci 15: 1094-1097, 2012.

35. Fukushima T, Tan X, Luo Y, Wang P, Song J, Kanda H, Hayakawa T, Kumagai T, Kakamu T, Tsuji M, et al: Heavy metals in blood and urine and its relation to depressive symptoms in Parkinson's disease patients. Fukushima J Med Sci 59: 76-80, 2013.

36. Zhao HW, Lin J, Wang XB, Cheng X, Wang JY, Hu BL, Zhang Y, Zhang $\mathrm{X}$ and Zhu JH: Assessing plasma levels of selenium, copper, iron and zinc in patients of Parkinson's disease. PLoS One 8: e83060, 2013.

37. Kumudini N, Uma A, Devi YP, Naushad SM, Mridula R, Borgohain R and Kutala VK: Association of Parkinson's disease with altered serum levels of lead and transition metals among south Indian subjects. Indian J Biochem Biophys 51: 121-126, 2014.

38. Hu Y, Yu SY, Zuo LJ, Piao YS, Cao CJ, Wang F, Chen ZJ, Du Y, Lian TH, Liu GF, et al: Investigation on abnormal iron metabolism and related inflammation in Parkinson disease patients with probable RBD. PLoS One 10: e0138997, 2015.

39. Costa-Mallen P, Zabetian CP, Agarwal P, Hu SC, Yearout D, Samii A, Leverenz JB, Roberts JW and Checkoway H: Haptoglobin phenotype modifies serum iron levels and the effect of smoking on Parkinson disease risk. Parkinsonism Relat Disord 21: 1087-1092, 2015.

40. Medeiros MS, Schumacher-Schuh A, Cardoso AM, Bochi GV, Baldissarelli J, Kegler A, Santana D, Chaves CM, Schetinger MR, Moresco RN, et al: Iron and oxidative stress in Parkinson's disease: an observational study of injury biomarkers. PLoS One 11: e0146129, 2016.

41. Mariani S, Ventriglia M, Simonelli I, Bucossi S, Siotto M, Donno S, Vernieri F and Squitti R: Association between sex, systemic iron variation and probability of Parkinson's disease. Int J Neurosci 126: 354-360, 2016. 